\title{
Implementação do diagnóstico da infecção pelo HIV para gestantes em Unidade Básica de Saúde da Família em Fortaleza, Ceará
}

\author{
HIV-testing for pregnant women in a Family Health Unit \\ in Fortaleza, Ceará
}

Maria Alix Leite Araújo ${ }^{1}$

N eiva Francenely Cunha Vieira ${ }^{2}$

Raimunda M agalhães da Silva ${ }^{1}$

${ }^{1}$ M estrado em Saúde Coletiva, Universidade de Fortaleza, UNIFOR.Av. Washington Soares 1321, Bairro Edson Queiroz. 60.811-905 Fortaleza CE. Alix.araujo@secrel.com.br ${ }^{2}$ Departamento de Enfermagem, Faculdade de Farmácia, Odontologiae Enfermagem, Universidade Federal do Ceará.
Abstract Free access to prenatal care and HIV tests represent the first step in the prevention of vertical transmission and guarantees early prophylactic measures. This study aims at analyzing the coverage of HIV testing in pregnant women in a Basic Family $\mathrm{H}$ ealth U nit in the city of Fortaleza, Ceará. The theoretical-methodological basis of the study was the grounded theory, which proposes generation of theory from data derived from the social reality. D ata were collected through participant observation, open interviews with physicians, nurses and pregnant women and in focal groups with pregnant women and health agents. The women faced institutional barriers to prenatal care and HIV testing. Difficulties in making theappointments for prenatal care hampered early identification of pregnant women and delayed their assistance. With respect to HIV testing, the study found that the Basic Health U nit does not collect the biologic material; there are few laboratories performing this kind of exam and those who do are very far from the pregnant women's residences. This study concludesthat even in theFamily Health U nit, the access to prenatal care for pregnant women remains difficult, and that the prevention of vertical HIV transmission depends on political, institutional and structural changes. Key words Accessibility, HIV infection, Primary health care, Family health, Pregnant women
Resumo A garantia do diagnóstico da infecção pelo HIV em gestantes representa a primeira etapa de prevenção da transmissão vertical. Falhas na cobertura desse diagnóstico impossibilitam a adoção precoce das medidas profiláticas. Esse estudo objetivou analisar a implementação da de tecção da infecção pelo HIV em gestantes em uma Unidade Básica de Saúde da Família em Fortaleza, Ceará. U tilizou referencial teórico-metodológico da grounded theory, que propõea construção de modelo teórico a partir dos dados da realidade social. Realizou-se observação participante e entrevistas abertas com médicos, enfermeiros, gestantes e grupos focais com gestantes e agentes de saúde. As gestantes enfrentam barreiras de acesso à consulta pré natal e ao teste anti-HIV. Evidenciaram-se dificuldades na marcação da consulta, na captação precoce da gestante e demora para atendimento. Quanto ao teste anti-HIV, detectou-se que a unidade básica não realiza a coleta do material biológico, poucos laboratórios o realizam e há distância geográfica entre os laboratórios e a residência da gestante. Concluiu-se que, mesmo em U nidades de Saúde da Família, o acesso à consulta pré-natal é difícil e a prevenção da transmissão vertical do HIV depende de mudanças políticas, institucionais e estruturais. Palavras-chave Acessibilidade, Infecção pelo H IV, Atenção primária à saúde, Saúde da Família, Gestantes 
Introdução

A epidemia de aids é um problema de grande magnitude que progride em todas as regiões do planeta. Ultimamente, dados epidemiológicos registram aumento de casos em mulheres, representando a possibilidade de transmissão vertical (TV) do HIV, que responde atualmente por mais de $80 \%$ dos casos de aids em crianças menores de 13 anos ${ }^{1}$.

0 aumento decasos deaids em mulheres exigiu a implementação de medidas concretas de saúde pública, como a realização de exames para o diagnóstico de infecção pelo HIV, doravante denominados testes anti-H IV, durante a gestação, acompanhada de aconselhamento, adoção universal da terapia anti-retroviral para gestantes e crianças expostas ao $\mathrm{HIV}^{2}$, medida esta que evitou cerca de 6.000 novos casos em crianças de 1997 a $2001^{3}$.

A garantia de realização do teste anti-HIV representa a primeira etapa da preven ção da TV, uma vez que é a partir do resultado, caso positivo, que se podem adotar as recomendações visando à diminuição da TV. Portanto, falhas na cobertura de testagem durante o pré natal é um fato grave, uma vez queimpossi bilita efetivamente a adoção precoce das medidas profiláticas.

0 alcance da meta de redução da TV do H IV dever-se-ia se fazer por meio do envolvimento da atenção básica, que representa a porta de entrada no sistema de saúde e normalmente local de procura da população para atendimento, especialmente mulheres grávidas para realizar 0 pré-natal.

Ocorre que o envolvimento da atenção básica com as ações de prevenção da TV do HIV tem acontecido de forma muito lenta. Treinamentos em aconsel hamento préepós-teste anti-HIV são realizados; entretanto, mesmo após os treinamentos, os profissionais ainda não conseguem inserir esta atividade na rotina dos serviços. Por outro lado, as unidades não apresentam recursos tecnológicos mínimos que garantam efetivamente a realização do teste (salas de coletas, organização do transporte das amostras para 0 laboratório e de recebimento dos resultados), 0 que pode estar repercutindo nas baixas coberturas de testagem em gestantes em algumas regiões do Brasil ${ }^{4}$.

Reconhece-se, portanto, que inserir ações de prevenção da TV do HIV na atenção básica representa um desafio, uma vez que, nesse contexto, a qualidade da aten ção pré-natal ainda deixa a desejar ${ }^{5,6}$. Portanto, essa propostaéuma das prin- cipais metas do Programa Nacional de DST/aids e esforços para o envolvimento da atenção básica vêm sendo desenvolvidos ao longo dos anos ${ }^{7,8}$.

Considerando-se as baixas coberturas de testagem do HIV no pré-natal, esse estudo tem por objetivo analisar a sua implementação em gestantes de uma Unidade Básica de Saúde da Família (UBASF) de Fortaleza, Ceará.

\section{M etodologia}

Trata-se de um estudo qualitativo que utilizou abordagem teórico-metodológica da grounded theory ${ }^{9}$, método construtivista que propõe uma análise indutiva, por meio da comparação constante das categorias, visan do à elaboração de um modelo teórico explicativo. Esse modelo, focado no processo de interação social, define que a coleta e análise dos dados devam ocorrer de forma concomitante.

0 trabalho decampo aconteceu em uma Unidade Básica de Saúde da Família (UBASF) da cidade de Fortaleza, Ceará, que se encontra na gestão plena da atenção básica à saúde e conta atualmente com 25 UBASF implantadas em áreas de risco.

A UBASF queparticipou do estudo conta com cinco equipes, acompanha em média 1.400 famílias e cadastrou, no ano de 2004, 274 gestantes ${ }^{10}$. Foi escolhida a partir dos seguintes critérios: estar solicitando o testeanti-HIV na rotina do pré natal, ter as equipes completas (médico, enfermeiro, auxiliar de enfermagem e agente de saúde) e disponibilidade dos profissionais para participar do estudo.

Os participantes do estudo

Na perspectiva da grounded theory, os participantes do estudo não são todos definidosa priori. 0 processo de coleta de dados inicia-se com pessoas ou grupos estratégicos e a definição dos próximos participantes éinfluenciada pelas categorias geradas dos resultados das primeiras entrevistas (theoretical sampling) 9 .

Inicialmente, essas categorias foram geradas das observações de consultas de pré-natal e de entrevistas com dois médicos e dois enfermeiros. $\mathrm{A}$ opção deincluir, primeiramente, médicos e enfermeiros, ocorreu por estes realizarem o atendimento pré natal, estarem treinados em aconsel hamento, solicitando e entregando o teste anti-HIV na rotina do atendimento. No decorrer do estudo, gestantes, agentes de saúde e a direção da unidade 
foram incluídos a partir das categorias que emergiram das análises dos primeiros dados ${ }^{9,11}$.

\section{Coleta de dados}

0 trabal ho de campo ocorreu durante os meses deagosto de 2004 a fevereiro de 2005 enão houve recusa de nenhum profissional para participar do estudo.

A coleta de dados iniciou-se com a observação partici pante e entrevistas abertas com médicos e enfermeiros. As entrevistas ocorreram em horários e dias combinados previamente.

As observações iniciais foram dos atendimentos pré-natais, para certificar-se de que realmente o exame anti-HIV estava sendo solicitado na rotina do serviço. As observações foram feitas no turno da manhã e uma das pesquisadoras permaneceu no consultório durante todo 0 atendimento pré natal.

Essas observações prosseguiram durantetodo período do trabalho de campo, ocorrendo livremente na unidade, atentando-se prioritariamente para o fluxo da gestante após a solicitação do teste e a coleta da amostra de sangue. As entrevistas abertas utilizaram como questão norteadora: "Fale-me como ocorre a testagem do HIV em gestantes nesta unidade". Ao longo das entre vistas, eram acrescidas outras indagações relacionadas à consulta e realização do exame.

A partir da codificação e categorização dos dados iniciais, foram selecionadas as primeiras categorias, o que definiu os próximos entrevistados: gestantes, agentes de saúde e o diretor da unidade. Com essas populações, trabalhou-se com grupos focais, cujos horários foram definidos a partir da facilidade de acesso às pessoas. $\mathrm{A}$ temática utilizada para a realização dos grupos focais estava relacionada à consulta prén natal eà realização do teste anti-H IV pela gestante, identificando suas facilidades e dificuldades.

Para a realização dos grupos focais, enviouse convite aos participantes com a anuência dos profissionais. 0 objetivo foi envolver, ao máximo, o trabal ho da pesquisa com as atividades da unidade e conseqüentementeter uma maior adesão dos participantes. As sessões duraram em torno deuma hora emeia eaconteceram em uma sala privativa da unidade.

Quando havia necessidade, outros esclarecimentos foram tomados com os profissionais já entrevistados, visando favorecer uma visão mais aprofundada dos achados e proporcionar maior validade às primeiras categorias.

0 trabalho de campo envolveu dez entrevis- tas individuais: quatro enfermeiros, três médicos, o gerente da unidade, três gestantes, quatro grupos focais: dois com gestantes e dois com agentes de saúde, dos quais participaram treze e doze pessoas, respectivamente.

Os dados da observação foram registrados no diário de anotações de campo e as entrevistas foram gravadas com a autorização dos sujeitos.

Visando não caracterizar os informantes, médicos e enfermeiros foram tratados pelo sexo masculino. O ptou-se por citar as entrevistas por ordem derealização e, no caso específico dos grupos focais, citar o participante (agente de saúde ou gestante). 0 diretor da unidade foi citado como "diretor".

\section{Organização dos dados para análise}

Na perspectiva da grounded theory $y^{9,11}$, a coleta eanálise dos dados ocorrem concomitante em um processo de interação e comparação constantes. U tiliza análise interpretativa das categorias para a elaboração de mapas conceituais, cujas estruturas são analisadas também externamente a partir da literatura pertinente.

A análise dos dados envolveu as seguintes etapas: codificação aberta, axial e seletiva. A codificação aberta consiste no processo de desmembramento, exame, comparação, conceitual ização e categorização.

$\mathrm{N}$ a codificação axial, ocorre o reagrupamento dos dados estabelecendo conexões entre as categorias, com o objetivo de expandir e aprofundar o modelo teórico. Nesta etapa, inicia-sea integração das categorias elaborando conexões com as subcategorias, visando identificar a variável central, processo principal da etapa de codificação axial.

A codificação sel etiva éa etapa de maior abstração e representa a finalização do processo de análise. Consiste no refinamento e integração final das categorias e subcategorias. 0 objetivo é identificar a variável central que deveser ampla e abstrata para incluir e expressar todas as outras categorias. Ao final do processo de codificação, definiu-se como, categoria seletiva, barreiras institucionais deacesso e, como subcategorias, acesso à consulta pré natal e acesso ao teste anti-HIV.

Durante o processo de codificação seletiva, 0 modelo teórico foi submetido à trajetória do método ou modelo de paradigma, que significa analisar os dados a partir das condições causais, fenômeno, contexto, condições intervenientes, estratégias de ação/interação e conseqüências ${ }^{11}$.

O projeto foi submetido ao Comitê de Ética 
da Universidade Federal do Ceará e obedeceu às orientações e normas éticas estabelecidas pela resolução no 196 do Conselho Nacional de Ética e Pesquisa.

\section{Resultados}

A coleta de dados envolveu 36 participantes cujas idades variaram de 20 a 55 anos ( 31 a 55 anos para os profissionais, 20 a 31 anos para as gestantes e 26 a 50 anos para os agentes de saúde).

Os profissionais médicos e enfermeiros trabalhavam entre dois e seis anos no Programa de Saúdeda Família. Entreas gestantes, sete haviam concluído o ensino fundamental, três tinham 0 ensino fundamental incompleto e três haviam concluído o ensino médio. Todas estavam com parceiro fixo na época das entrevistas. Os agentes de saúdetinham detrês meses a cinco anos de atuação no PSF enenhum havia recebido treinamento em DST/HIV/aids.

\section{Barreiras institucionais de acesso}

Constatou-se que as gestantes enfrentam muitas barrei ras institucionais de acesso ao teste anti-HIV eestas ocorrem devido à dificuldadede marcação da consulta pré-natal e para a realização do teste anti-HIV.

N esse estudo, considera-se como barreiras institucionais de acesso todas as condições diretas ou indiretas que dificultavam a realização do testepela gestante.

\section{Dificuldade de acesso à consulta pré-natal}

A dificuldade de acesso das gestantes à consulta prénatal foi definida como: situações que comprometem a marcação da consulta, déficit na captação precoce da gestante na comunidade e não priorização do atendimento à gestante.

I dentificou-se que as gestantes não podem se dirigi r diretamenteà unidade para marcar a consulta pré natal. Esta marcação tem que ocorrer por intermédio do agente de saúde, o que tem tornado difícil o acesso, pois estes profissionais na grande maioria das vezes demoram a dar um retorno.

As gestantes relataram que precisam ser muito insistentes para conseguir fazer o pré-natal, fato demonstrado nos depoimentos abaixo:

Para marcar a consulta do prénatal tem que ser com o agente de saúde. Aí demora muito para elas poderem deixar o papel em casa. (gestante)
Mas como eu fui muito insistente, né, eu fui muito insistente e corri e fui atrás, eu corri atrás. Se a pessoa não correr atrás não consegue nada, se você não se esforçar, se você não for assim uma pessoa muito insistente você não vai conseguir nada não. (gestante)

De acordo com esses depoimentos, as usuárias se sentem prejudicadas e sem autonomia para procurar a unidade, pois a marcação das consultas dependedo encaminhamento do agente de saúde. Por outro lado, não existe um sistema organizado na unidade para captação precoce da gestante na comunidade, o que com certeza está contribuindo para retardar o início das consultas pré-natais e, conseqüentemente, da realização do teste anti-HIV.

Eu acho que os agentes de saúde poderiam captar precocemente a gestante, mas a gentecapta tardiamente equando recebe [o resultado] ela já pariu. I sso vem acontecendo muito. (M édico 1 ).

Ocorrendo em uma unidade de saúde da família, essa situação causa estranheza, uma vez que a implantação dessa estratégia visa exatamente reverter o modelo de atenção em saúde excludente praticado no Brasil. Tal dificuldade está relacionada à forma de organização do serviço, que não prioriza os atendimentos de prénatal, comprometendo 0 acesso precoce da gestante e conseqüentemente a realização do teste anti-HIV. De acordo com os depoimentos abaixo, a dificuldade para marcar a consulta é uma realidade, levando a gestante a ter que chegar à unidade de madrugada ou, ainda, a desistir de realizar o prénatal na referida unidade.

Para marcar a ficha aqui é muito difícil, mesmo estando grávida. (gestante)

A minha área tem poucas gestantes sen do acompanhadas aqui. Eu acho que é devido a demora para 0 atendimento. (agente de saúde)

M uita coisa tinha que ser modificada para ser mais prático para a paciente. Elas têm que chegar demadrugada. (enfermeiro 3 )

No que diz respeito à prevenção da transmissão vertical do HIV, essa situação égrave, uma vez que, iniciando o prénatal tardiamente, as gestantes que porventura tiverem o resultado positivo não iniciarão a quimioprofilaxia no tempo preconizado pelo M inistério da Saúde, podendo trazer danos para o bebê. Também não terão a oportunidade de receber apoio emocional, tão importante para as gestantes nessa situação. 
Dificuldades de acesso ao teste anti-HIV

A análise das dificuldades de acesso ao teste anti-HIV inclui todos os aspectos que dificultavam ou retardavam a realização do teste pela gestante, a saber: escassez de laboratórios que realizam o exame anti-HIV, falta de priorização dos exames de rotina, baixas con dições financeiras das gestantes e fal ta de posto de coleta na unidade.

Após a primeira consulta de prénatal e de posse da requisição do exame, a gestante enfrenta muitos obstáculos para realizar o teste antiHIV. O primeiro deles é a sua marcação. Todos os exames de rotina são marcados na sala da direção, por intermédio do sistema informatizado, que está conectado a laboratórios públicos e conveniados à prefeitura municipal. 0 laboratório central municipal de saúde pública é o único que realiza o teste anti-HIV e os laboratórios particulares conveniados realizam os ou tros exames de rotina de pré-natal (hemograma, VDRL, sumário de urina, etc.), exceto o anti-HIV.

Um dos aspectos relacionados à barreira de acesso ao exame anti-HIV está na escassez de laboratórios, gerando sobrecarga no laboratório central, considerando que este realiza todos os testes anti-HIV solicitados nas unidades da Secretaria M unicipal de Saúde de Fortaleza.

O sistema informatizado com prontuário eletrônico foi implantado pela prefeitura municipal de Fortaleza em todas as unidades de saúde há mais ou menos dois anos e está conectado aos laboratórios. Todas as consultas e solicitações de exames das gestantes só podem ser realizadas por intermédio deste sistema. O s exames são marcados na própria unidade e as gestantes devem se dirigir aos laboratórios na data estabelecida, para colher o material biológico. O correque todos os exames do HIV são marcados em um único laboratório, sobrecarregando-0, como ilustram os trechos abaixo:

Eu não fiz o teste ainda porque não tem vaga ainda no laboratório. Eu acho que eu vou perder [o prazo da requisição], porque quando eu for para a consulta agora no dia 23 é para levar 0 resultado, como é que eu vou levar se eu nem fiz ainda? (gestante)

Só que infelizmente dentro da organização ge rencial da prefeitura, o queaconteceu équequando entra a solicitação [do exame] no sistema, a paciente ela entra numa fila de espera. (enfermeiro 2)

Por outro lado, quando conseguem marcar os exames, estes são colocados para vários laboratórios diferentes, obrigando as mulheres a se dirigir, várias vezes, a estes laboratórios para con- seguir realizá-los. Durante o trabalho de campo, houve a oportunidade de assistir a vários momentos de marcação dos exames e observou-se que, mesmo o profissional tendo interesse em colocá-los todos para o mesmo laboratório, tornava-se impossível, pois o próprio sistema limitava devido à indisponibilidade de laboratórios que realizam o anti-HIV.

Quando ela tem a possi bilidade de fazer o exame, ele é marcado nos laboratórios de Fortaleza como um todo, quetenha a vaga na data ena hora que tiver explicitado no sistema. (enfermeiro 2)

Demorei em fazer o teste por problemas aqui no posto mesmo. A gentevai marcar, tem que esperar. Fiz lá no centro de especialidades. Eu fiz al guns exames lá e outros em um hospital. (gestante 3)

Então, por ocasião da marcação dos exames do pré-natal, o sistema automaticamente agenda os anti-HIV para o laboratório central municipal e os outros exames são marcados em diferentes laboratórios conveniados de acordo com a disponibilidade de vagas. Essa situação, além de gerar sobrecarga no laboratório municipal e conseqüentemente atraso na marcação e entrega do resultado, pode fazer com que as gestantes não realizem o teste.

A dificuldaderesidena marcação dos exames de rotina do pré natal em diferentes laboratórios do município, o que obriga as gestantes a dirigir-se a vários locais, dando a perceber que os exames de rotina do pré-natal não são priorizados. As observações descritas foram reforçadas pelos profissionais:

Os exames são marcados em locais diferentes e isso dificulta. (enfermeiro 1)

Elastêm uma dificuldade muito grande porque o sistema, ao marcar os exames, marca para locais diferentes, então tipo assim: marcam o VDRL para um lugar, o anti-HIV para outro lugar, hemograma em outro lugar, sumário de urina em outro lugar e daí a gestante tem que ir a vários locais para fazer o exame. (médico 1 )

A paciente, ela tem quefazer hojeuma via crucis. Ela faz uma peregrinação entre os laboratórios para realizar esses exames. (enfermeiro 2)

As observações de campo permitiram comprovar a indisponibilidade de laboratórios para realizar o exame anti-HIV, o que dificulta sobremaneira 0 acesso das gestantes ao teste.

Vale ressaltar também que as UBASFs normalmente se localizam em áreas de risco, onde residem pessoas de baixo poder aquisitivo, que não dispõem de recursos financeiros suficientes. 0 fato de ter que se deslocar a vários laboratórios pode fazer com que muitas mulheres deixem 
de realizar os exames por falta de condições financeiras, o que deve ser considerada uma situação grave e que compromete a qualidade da assistência pré-natal. Os depoimentos dos diferentes sujeitos descritos a seguir corroboram com essas afirmações:

Eu deixei de fazer [o teste], porque eu tinha que ir para dois cantos. Pesa no bolso da gente. (gestante)

Os meus exames estão marcados todos em locais diferentes e dias diferentes. Tomara que ela não bote muito para longe, porque para longe é muito difícil ir pois eu tenho que pegar ônibus. (gestante)

A demora na marcação dos exames, aliada à falta de condições financeiras das mulheres, estão contribuindo para as baixas coberturas de testagem do HIV no pré-natal. Podemos acrescentar a todos estes aspectos anteriormente descritos a indisponibilidade de postos de coleta de sangue nas U BASFs e de mais laboratórios equipados para leitura das amostras. Ocorre que 0 fato de ter que colher os exames de rotina do pré- natal em locais diferentes pode fazer com que as gestantes coloquem em segundo plano a realização do teste anti-HIV.

Por uma determinação interna da unidade, os resultados de todos os outros exames, com exceção do anti-HIV, retornam pelo sistema e estão disponíveis geralmente no dia agendado para 0 atendimento de retorno. Segundo a direção, essa medida foi tomada devido à dificuldade de guardar sigilo do resultado, apesar do acesso aos prontuários el etrônicos serem restritos aos médicos eenfermeiros. Abaixo os depoimentos esclarecem tais situações:

Todos os exames vêm pelo sistema, só não 0 anti-HIV. $O$ anti-HIV a paciente vai lá [laboratório central] e recebe. Não vem pelo sistema, não pode. Se viesse pel o sistema não tinha sigilo. Se der negativo tudo bem, mas se der positivo? (diretor da unidade)

\section{Discussão}

Evidenciou-sequeas mulheres grávidas enfrentam muitas dificuldades para realizar o teste anti-HIV, devido às barreiras de acesso institucional, muitas delas provocadas pela própria organização interna da unidade, 0 chamado acesso funcional ${ }^{13}$.

0 acesso aos serviços de saúde é um desafio para a efetiva implementação do Sistema Ú nico de Saúde, caracterizando-se como um dos principais problemas enfrentados atualmente pelo poder público. No Brasil, estudos evidenciam diferenças sociais de acesso nas diversas regiões do país e nos diferentes segmentos populacionai $5^{14 \cdot 16}$ e apontam um cenário desalentador.

0 fato da marcação da consulta ser realizada através dos agentes de saúde provoca dificuldade de acesso e falta de agilidade na captação de gestantes, situação que gera insatisfação da usuária diante da demora para a marcação da consulta. Atuar em área adstrita deveria favorecer ao PSF o estabelecimento do vínculo com a comunidade, situação que poderia facilitar um acesso mais precoce da gestante.

Causa surpresa a existência dessa queixa, haja vista que cabeao agente de saúde visitar no mínimo uma vez por mês cada família da sua área de abrangência, identificando as gestantes e encaminhando ao pré-natal o mais precocemente possível ${ }^{17}$.

Constatou-se, portanto, que não existe indisponibilidade de fichas para consultas e nem carência de profissionais médicos para o atendimento, principais motivos relacionados com a dificuldade de acesso eutilização dos serviços de saúde no Brasil ${ }^{18}$. Observa-se que o problema está relacionado à forma de organização na assistência pré-natal.

Considerando que a atenção pré natal éuma das prioridades do PSF e tem a finalidade de detectar precocemente os fatores de risco para anomalias ou enfermidades nas mulheres grávidas, visando prestar intervenções eficazes e oportunas, 0 acesso à consulta deve ser uma prioridade, especialmente neste programa, e o programa de saúde da família se apresenta como um espaço estratégico cujo envolvimento é imprescindível para que a política de prevenção da TV realmentese concretize.

Estudo com gestantes HIV positivas em Caxias do $\mathrm{Sul}^{19}$ mostrou que as mulheres não haviam realizado prénatal ou este não foi iniciado precocemente, perdendo-sea melhor época para início do tratamento. Esses achados reforçam a falta de captação precoce da gestante e a dificuldade de acesso à consulta pré-natal.

Os resultados deste estudo indicam que as barreiras de acesso da gestante à consulta pré natal e ao teste anti-H IV podem gerar desgaste emocional, pela necessidade da persistência, pela espera e a grande possibilidade de não conseguir realizar o teste ou de não receber o resultado a tempo. Enquanto isso, a gravidez segue o seu curso, podendo tornar o tempo escasso para atingir a meta de prevenção, controle e tratamento do HIV em mulheres grávidas. 
U sando como justificativa a dificuldade de manutenção do sigilo dos testes, os técnicos determinaram quea gestante deveria ir receber pessoalmente no laboratório municipal o resultado do exame anti-HIV. Essa decisão não foi dialogada com as usuárias e aumentou com certeza a dificuldade de acesso, pois algumas mulheres deixam de ir receber o resultado do exame antiHIV devido à falta de condições financeiras.

0 resultado do teste anti-HIV é realmente um exame sigiloso, assim como todos os outros exames das gestantes. Internamente, as equipes deveriam discutir e encontrar mecanismos que garantissem absoluto sigilo dos resultados, independentemente de positivo ou negativo. Vale salientar que 0 acesso aos prontuários é restrito aos profissionais, só podendo ocorrer utilizando-se senha individual, o que deveria assegurar o sigilo do resultado do teste, não justificando assim tal medida.

A organização técnico-administrativa desta unidade de saúderepercutena forma de prestação dos serviços à comunidade, dificultando o acesso da mulher grávida infectada pelo HIV às medidas de prevenção da transmissão vertical do HIV.

$O$ que se observou na UBASF quanto às indefinições de prioridades no atendimento às gestantes não atende as recomendações da proposta do PSF, pois com promete a qualidade do atendimento e as respostas às demandas da comunidade. 0 acompanhamento pré-natal é uma pri- oridade no PSF, mas na prática não se configura como tal, repercutindo na qualidade da atenção prestada a gestante.

\section{Conclusão}

Pode-se concluir que o problema de acesso da gestante ao teste anti-HIV precisa ser considerado do ponto de vista não só da disponibilidade do exame propriamente dito, mas em toda estruturação do cuidado dispensado a esta mulher durante o pré-natal na atenção básica.

Uma medida sugerida que poderia minimizar tal situação é efetivamente priorizar o agendamento das consultas e do exameanti-HIV desta clientela, visto que a gestação tem um tempo limitado e exigeurgência na realização das medidas profiláticas que visam prevenir o risco de contaminação do bebê, bem como o acompanhamento com apoio emocional à mulher HIV positiva.

Todos esses fatores relacionados às barreiras institucionais de acesso são considerados importantes para a prevenção da TV do HIV e necessitam ser superados, uma vez que devem estar contribuindo para baixas coberturas de testagem do HIV durante o pré-natal, impossibilitando uma assistência de qual idade à gestante, além de agravar ainda mais o quadro de morbi-mortalidade materno-infantil em Fortaleza, Ceará.

\section{Colaboradores}

M AL Araújo, NFC Vieira e RM da Silva participaram igualmente de todas as etapas da elaboração do artigo. 


\section{Referências}

1. Brasil. M inistério da Saúde. Boletim Epidemiológico de Aids e DST 2004; 1(1).

2. Brasil. Ministério da Saúde. Programa Nacional de DST/Aids. Recomendações para profilaxia da transmissão vertical do HIV e terapia anti-retroviral em gestantes. Brasília: Ministério da Saúde; 2004.

3. Brito AM, Sousa JL, Luna CF, Dourado I. Tendência da transmissão vertical de aids após terapia antiretroviral no Brasil. Rev. Saúde Pública 2006; 40(Supl):18-22.

4. Souza Júnior PR, Szwarcwald CL, Barbosa Júnior A, Carvalho M F, Castilho EA. Infecção pelo HIV durante a gestação: estudo-sentinela parturiente, Brasil, 2002. Rev. Saúde Pública 2004; 38(6):764-772.

5. Coimbra LC, Silva AAM, Mochel EG, Alves MTS$\mathrm{SB}$, Ribeiro VS, Aragão VM F, Bettiol H. Fatores associados à inadequação do uso da assistência pré natal. Rev. Saúde Pública 2003; 37(4):456-462.

6. Moura ERF, Holanda Jr F, Rodrigues M SP. Avaliação da assistência pré-natal oferecida em uma microrregião de saúde do Ceará, Brasil. Cad Saúde Pública 2003; 19(6):1791-1799.

7. Brasil. M inistério da Saúde. A conselhamento em DST/HIV/Aids para a atenção básica. Brasília: M inistério da Saúde; 2003.

8. Brasil. M inistério da Saúde. Secretaria de Atenção à Saúde. Departamento de Ações Programáticas Estratégicas. Política nacional de atenção integral à saúde da mulher: plano de ação 2004-2007. Brasília: Ministério da Saúde; 2004. 48 p.

9. Strauss AL. Corbin J. Grounded theory methodology: an overview. In: Denzin NK. Lincoln YS, editors. $\mathrm{H}$ andbook of qualitative research. Thousand Oaks, CA: Sage; 1994.

10. Prefeitura Municipal de Fortaleza. Secretaria Municipal de Saúde. Programa de Saúde da Família (PSF). Boletim de Saúde de Fortaleza 2002; 6(3).

11. Chamaz K. Grounded theory: objectivist and constructivist methods. In: Denzin NK. Lincoln YS, editors. Strategies of qualitative inquiry. London: Sage Publication; 2003.
12. Brasil. Resolução no 196/96, de 10 de outubro de 1996. Dispõe sobre diretrizes e normas técnicas regulamentadoras de pesquisas envolvendo seres humanos. Brasília, 1996. Diário Oficial da União 1996; 16 out.

13. Unglet CVS. Territorialização em sistemas de saúde. In: M endes EV, organizador. Distrito sanitário. São Paulo: Hucitec; Rio de Janeiro: ABRASCO; 1995. p. 221-235.

14. Araújo $M A L$, Leitão $G C M$. Acesso à consulta a portadores de Doenças Sexualmente Transmissíveis: experiências de homens em uma unidade de referência em Fortaleza, Ceará. Cad Saúde Pública 2005; 21(2):396-403.

15. Lima AJC, Azory EB, Bastos LHC, Coutinho MM, Pereira NN, Ferreira SCC. Desigualdade no acesso e utilização de serviços de saúde no Brasil. Saúde em Debate 2002; 26(60):62-70.

16. Ramos DD, Lima MAS. Acesso e acolhimento aos usuários em uma unidade de saúde de Porto Alegre, Rio Grande do Sul, Brasil. Cad Saúde Pública 2003; 19(1):27-34.

17. Brasil. M inistério da Saúde. Secretaria de Políticas de Saúde. Departamento de Atenção Básica. 0 trabalho do Agente Comunitário de Saúde. Brasília: M inistério da Saúde; 2000.

18. Assis MMA, Villa TCS, Nascimento MAA. Acesso aos serviços de saúde: uma possibilidade a ser construída na prática. Cien Saude Colet 2003; 8(3):815823.

19. Stefani M, Araújo BF, Rocha N M P. Transmissão vertical do HIV em população de baixa renda do Sul do Brasil. DST. J bras Doenças Sex Transm 2004; $16(2): 33-39$.

Artigo apresentado em 14/04/2007

Aprovado em 17/08/2007

Versão final apresentada em 18/09/2007 